\title{
Paying for Minimum Interest Rate Guarantees Who Should Compensate Who?
}

\author{
Astrup Jensen, Bjarne; Sørensen, Carsten
}

Document Version

Final published version

Publication date:

2000

License

CC BY-NC-ND

Citation for published version (APA):

Astrup Jensen, B., \& Sørensen, C. (2000). Paying for Minimum Interest Rate Guarantees: Who Should Compensate Who? Institut for Finansiering, Copenhagen Business School. Working Papers / Department of Finance. Copenhagen Business School No. 2000-1

Link to publication in CBS Research Portal

\section{General rights}

Copyright and moral rights for the publications made accessible in the public portal are retained by the authors and/or other copyright owners and it is a condition of accessing publications that users recognise and abide by the legal requirements associated with these rights.

Take down policy

If you believe that this document breaches copyright please contact us (research.lib@cbs.dk) providing details, and we will remove access to the work immediately and investigate your claim. 
WP 2000-1

Paying for minimum interest rate guarantees: Who should compensate who?

af

Bjarne Astrup Jensen \& Carsten Sørensen

INSTITUT FOR FINANSIERING, Handelshøjskolen i København

Solbjerg Plads 3, 2000 Frederiksberg C

tlf.: 38153615 fax: 38153600

DEPARTMENT OF FINANCE, Copenhagen Business School

Solbjerg Plads 3, DK - 2000 Frederiksberg C, Denmark

Phone (+45)38153615, Fax (+45)38153600

www.cbs.dk/departments/finance

ISBN 87-90705-32-7

ISSN 0903-0352 


\title{
Paying for minimum interest rate guarantees: Who should compensate who?
}

\author{
Bjarne Astrup Jensen and Carsten Sørensen* \\ JEL Classification: G11,G13
}

This version:

12th January 2000

First version: December 1998

${ }^{*}$ Department of Finance, Copenhagen Business School, Solbjerg Plads 3, DK-2000 Frb. C., Denmark. e-mail: fibj@cbs.dk and ficso@cbs.dk, respectively. We thank for comments on earlier versions of the paper from J. Aa. Nielsen and from participants at Danske Bank Symposium on Embedded Options, French Finance Association Annual Meeting 1999 and a seminar at Department of Finance, Copenhagen Business School. The authors gratefully acknowledge financial support from the Danish Social Science Council. 


\begin{abstract}
Defined contribution pension schemes and life insurance contracts often have a minimum interest rate guarantee as an integrated part of the contract. This guarantee is an embedded put option issued by the institution to the individual, who is forced to hold the option in the portfolio. However, taking the inability to short this saving and other institutional restrictions into account the individual may actually face a restriction on the feasible set of portfolio choices, hence be better off without such guarantees. We measure the effect of the minimum interest guarantee constraint through the wealth equivalent and show that guarantees may induce a significant utility loss for relatively risk tolerant investors.

We also consider the case with heterogenous investors sharing a common portfolio. Investors with different risk attitudes will experience a loss of utility by being forced to share a common portfolio. However, the relatively risk averse investors are partly compensated by the minimum interest rate guarantee, whereas the relatively risk tolerant investors are suffering a further utility loss.
\end{abstract}

Keywords: Minimum interest rate guarantee, asset allocation restrictions, utility loss, wealth equivalent, heterogenous investors. 


\section{Introduction}

Pension savings in countries with a considerable weight of funded pension schemes are often of a mandatory nature with savings plans related to labour market contracts.

In some countries such funded pension schemes, operating on an actuarial reserve basis, are also required by law to have a minimum interest rate guarantee which ensures a minimum growth rate of the individual pension saver's reserves. This growth rate may be annual or may be guaranteed as an average over long time intervals. In this paper we only consider the case with the guarantee as an average over a given time horizon.

The point of view in the existing literature is that an insurance policy or a pension plan equipped with a minimum interest rate guarantee provides the buyer with a useful guarantee. The seller is issuing a put option enabling the buyer to receive a minimum guaranteed rate of return in cases where the return on the underlying investment falls short of this guaranteed rate of return. On the other hand the buyer receives the return of the underlying investment whenever its return exceeds this minimum.

The literature on interest rate guarantees has mainly focused on the pricing of the implicit put option provided by the guarantor. Early examples are Brennan and Schwartz $(1976,1979)$, whereas more recent examples are found in e.g. Bacinello and Ortu (1993a,b), Nielsen and Sandmann $(1995,1996)$ and Aase and Persson (1997).

A related topic is the valuation of the surrender option in policies allowing the investor to exit prematurely at a cost. This can be of interest whenever the accumulated wealth cum guarantee exceeds the actual market value of the underlying portfolio. See e.g. Albizzati and Geman (1994) and Grosen and Jørgensen $(1997,1999)$.

However, the pricing of the guarantee cannot be done without an explicit assumption on the investment policy followed by the guarantor, and this investment policy will itself depend upon the existence of an interest rate guarantee. In fact, the investor may be in a position where this minimum interest rate guarantee is against what he or she would have wanted from a utility maximization point of view. The only possible response to a more and more binding constraint is to switch away from risky investments and into risk-free positions in the bond market. Hence we consider the interest rate guarantee as a restriction on the permissible portfolio strategies applicable to the pension fund contributions. In cases where the institutional saving constitutes a major part of the savings of individuals, and where this saving in part or in full may be required by law or somehow have a mandatory character, this can actually be a binding constraint on the overall asset allocation problem.

We will pursue the following analysis under the assumption of dynamically complete markets as far as the pricing of financial assets is concerned. In practice it may be difficult for the individuals to circumvent the effects of restrictions on institutional asset allocation decisions, which is partly due to the fact that such savings cannot be put up as collateral in order to undertake other offsetting financial positions. Hence in the paper we do not allow any given individual to trade in financial assets on her own account outside the pension scheme.

The specific utility optimization problem in this paper only has a consumption objective at the horizon. This is assumed in order to keep the analysis at the simplest possible level, but it can be extended without changing the basic points of the paper. We are choosing an analytically tractable class of utility functions in such a manner that problems with negative wealth positions 
automatically will be avoided. The menu of financial assets in the underlying economic model consists of $N$ risky securities and a bank account.

The unrestricted optimization problem is solved analytically. ${ }^{1}$ The solution to the restricted optimization problem with a minimum interest rate guarantee corresponds to the following portfolio insurance policy:

1. Invest $x W_{0}$ in the same way as what is optimal with no constraints, and

2. buy a put option up front at a premium, which is a fraction $(1-x) \in(0,1)$ of initial wealth $W_{0}$, and with

- the unrestricted portfolio $x W_{0}$ as the underlying asset and

- the wealth level $W_{0} e^{g T}$, guaranteed by the interest rate guarantee, as the exercise price

The fraction $x$ is found numerically as the solution to one equation with one unknown, reflecting that the investment in the portfolio must equal initial wealth. If the appropriate put option which ensures the fulfilment of the interest rate guarantee is not readily available in the market, it can be duplicated through a dynamic self-financing trading strategy starting from $(1-x) W_{0}$.

The solution generalizes the optimal strategies in related papers on portfolio insurance, e.g. Rubinstein (1985), Basak (1995) and Grossman and Zhou (1996). In particular Basak (1995) and Grossman and Zhou (1996) are concerned with a characterization of market equilibrium and endogenously derived equilibrium asset price processes in the presence of "floor constraints", whereas the present analysis takes the market price processes as a given input to any particular individual's optimization problem.

In the paper we analyze the utility loss of imposing a minimum interest rate guarantee as an exogenous constraint on the investor's ability to tailor her portfolio. Formally we apply the concept of a wealth equivalent, i.e. the magnitude of initial wealth that with no constraints gives the investor the same level of expected utility as that obtainable with her given initial wealth, but with constraints on the asset allocation decision. This is analogous to the certainty equivalent in expected utility analysis and is used here as the measure of the investor's aversion to the interest rate guarantee constraint. ${ }^{2}$ We derive the comparative statics of this wealth equivalent towards changes in the level of the guarantee and changes in the relative risk aversion. Furthermore, we numerically demonstrate the effects of the asset allocation restriction for the classical Black-Scholes model with a constant interest rate and for the Black-Scholes model combined with a Vasicek term structure model. It turns out that the multiplicity of assets and a possible stochastic interest rate add very little. The parameters of primary importance are the volatility of the pricing kernel and the yield to maturity on the zero coupon bond expiring at the investment horizon. To the extent that these parameters do not change with a change in the menu of assets the wealth equivalent is unaffected, although the optimal portfolio policy will obviously change as a response to a change in the menu of assets. For realistic parameter values

\footnotetext{
${ }^{1}$ A related analysis of fixed income portfolio management, using a similar technique for a special case of the more general model in this paper, is found in Sørensen (1999).

${ }^{2}$ This idea of measuring the effect of a suboptimal asset allocation decision has been used by other authors in different contexts, see e.g. Ang and Bekaert (1999), Brennan, Schwartz and Lagnado (1997), Campbell and Viceira $(1998,1999)$ and Das and Uppal (1996).
} 
the results suggest that imposing a minimum interest rate guarantee may induce a significant utility loss for relatively risk tolerant investors.

Another feature of mandatory savings plans and other institutionalized collective savings plans analyzed in the paper is the effect of the minimum interest rate guarantee when investors with different risk attitudes are pooled in a pro rata shared common portfolio. We provide analytical and numerical results for this situation as well. Investors with different risk attitudes will experience a loss of utility by being forced to share a common portfolio, because the fund manager must compromise between the preferences of the members in an investment pool. It turns out that this effect can be significant per se. However, when an interest rate guarantee is introduced, investors with high levels of relative risk aversion are compensated partly for the loss induced by an "aggressive" investment policy, whereas investors with a low level of relative risk aversion are suffering a further utility loss relative to the loss induced by a "conservative" investment policy.

The paper is organized as follows. In section 2 the investment problem is set up and the solutions to the unrestricted and restricted problems are derived. In section 3 we derive comparative statics of the wealth equivalent with respect to the level of the interest rate guarantee as well as the level of relative risk aversion. In section 4 we present numerical examples. The analysis is extended to the case with heterogenous agents sharing a common portfolio in section 5 . Section 6 concludes the paper. The details of proofs and other technicalities of the modelling framework are to a large extent carried out in the appendices of the paper.

\section{The investment problem}

Consider an investor with initial wealth $W_{0}$ and investment horizon $T$. The investor's objective is assumed to be expected utility maximization w.r.t. the accumulated wealth at the time horizon $T$. The investor's utility function is assumed to belong to the class of constant relative risk aversion (CRRA) utility functions:

$$
U\left(W_{T}\right)=\frac{W_{T}^{1-\gamma}}{1-\gamma}, \quad \gamma>0
$$

including the logarithmic utility function $U\left(W_{T}\right)=\log \left(W_{T}\right)$ for $\gamma=1 .^{3}$

There is no utility attached to intermediate consumption. This investor can invest in a combination of

1. an asset with a locally risk-free return $r_{t}$. The infinitesimally risk-free interest rate $r_{t}$ is allowed to vary stochastically, but only within the class of Gaussian term structure models

and

\footnotetext{
${ }^{3}$ The usual way of representing these preferences in order to get the logarithmic utility function as a limiting case is as $U\left(W_{T}\right)=\frac{W_{T}^{1-\gamma}-1}{1-\gamma}$. However, this is just an addition of a constant of no consequence for preference representation, but involving a more complicated notation elsewhere.
} 
2. a menu of $N$ assets with locally risky returns and price processes $P_{t}=\left(P_{t}^{1}, P_{t}^{2}, \ldots, P_{t}^{N}\right)$ :

$$
d P_{t}=\left(\operatorname{diag}\left[r_{t} 1_{N}+V \lambda\right] \cdot P_{t}\right) d t+\operatorname{diag}\left[P_{t}\right] \cdot V \cdot d Z_{t}
$$

where

- $V$ is an $N \times M$-matrix of diffusion coefficients. These are denoted as $\sigma_{i j}, i=1,2, \ldots, N$, $j=1,2, \ldots, M$, and fulfil the usual conditions required for the stochastic processes to be well-defined

- $Z_{t}$ an $M$-dimensional standard Brownian motion

- $\lambda$ an $M$-vector of deterministic risk-premia

- $1_{N}$ is an $N$-vector of ones

For an individual asset, say $P_{t}^{j}$, the price process becomes

$$
\frac{d P_{t}^{j}}{P_{t}^{j}}=\left(r_{t}+\sum_{m=1}^{M} \lambda_{m} \sigma_{j m}\right) d t+\sum_{m=1}^{M} \sigma_{j m} d Z_{t}^{m}
$$

It is well-known that $V$ - without loss of generality - can be chosen as a lower triangular matrix if so desired for analytical or computational purposes. Different models arise due to different specifications of the menu of assets, the number of risk-factors and the character of the interest rate process.

$\lambda$ is assumed to be constant, but the results are easily modified to encompass a time-varying, deterministic function $\lambda(t)$. For notational reasons this is not stated explicitly here.

In a standard probabilistic setup, $\left(\Omega, \mathcal{F}, \mathcal{P},\left\{\mathcal{F}_{t}\right\}_{t=0}^{t=T}\right)$, the filtration $\left\{\mathcal{F}_{t}\right\}_{t=0}^{t=T}$ is taken as the filtration generated by $Z_{t}^{m}, m=1,2, \ldots, M$. The market is assumed dynamically complete by construction, i.e. the rank of $V$ is $M$. Hence the pricing kernel or state price density, denoted by $M_{t}$, is uniquely determined, and it has the usual properties:

- $M_{0}=1$

- For any asset with price process $P_{t}^{j}$ the process $M_{t} P_{t}^{j}$ is a martingale, i.e. $P_{t}^{j}=E_{t}\left[\frac{M_{T}}{M_{t}} P_{T}^{j}\right]$

- In particular, the process $M_{t} e^{\int_{0}^{t} r_{s} d s}$ is a martingale

The pricing kernel $M_{t}$ is known to be the inverse of the optimal growth portfolio chosen by an investor with a logarithmic utility function, $U\left(W_{T}\right) \equiv \log W_{T} \cdot{ }^{4}$ It is the solution to the following stochastic differential equation:

$$
d M_{t}=-r_{t} M_{t} d t-M_{t} \lambda^{\prime} d Z_{t}, \quad M_{0}=1
$$

Or, alternatively, in integral form:

$$
M_{t}=e^{-h_{t}-\lambda^{\prime} Z_{t}-\frac{1}{2}\|\lambda\|^{2} t}=e^{-\left(h_{t}+\lambda^{\prime} Z_{t}\right)-\frac{1}{2}\|\lambda\|^{2} t}
$$

\footnotetext{
${ }^{4}$ See e.g. chapter 6 in Merton (1992) or Duffie (1996), chapter 6.
} 
where $h_{t}$ is defined by

$$
h_{t} \equiv \int_{0}^{t} r_{s} d s
$$

Some immediate consequences of the restrictions on the model parameters are stated in the following proposition.

Proposition 1 For a Gaussian interest rate process $r_{t}$ and constant (deterministic) market prices of risk $\lambda$ :

1. the accumulation factor $e^{h_{T}}$ is either deterministic or log-normally distributed

2. $M_{T}$ is log-normally distributed. Hence it can be represented as

$$
M_{T} \equiv \exp \left\{\mu_{M_{T}}-\frac{1}{2} \sigma_{M_{T}}^{2}+\sigma_{M_{T}} \mathcal{N}\right\}
$$

where $\mathcal{N}$ is a $N(0,1)$-variable and $\sigma_{M_{T}}=\sqrt{\operatorname{var}_{0}\left(h_{T}+\lambda^{\prime} Z_{T}\right)}$

3. the zero coupon bond price or discount factor $D(0 ; T)$ and the associated zero coupon interest rate $y(0 ; T)$ at time zero is given by

$$
D(0 ; T) \equiv \exp [-T y(0 ; T)]=E_{0}\left[M_{T}\right]=\exp \left\{\mu_{M_{T}}\right\}
$$

The choice of the CRRA class of utility functions is analytically convenient. As will become clear in the next section, the optimally invested wealth $W_{T}$ as well as the kernel-weighted optimal wealth $M_{T} W_{T}$ become log-normally distributed. This enables the calculation of analytical solutions and sensitivity analysis with respect to the relative risk aversion parameter $\gamma$. Being log-normally distributed we are also sure that the optimally invested wealth is always positive, i.e. the investor automatically satisfies an implicit solvency constraint. ${ }^{5}$

\subsection{Optimal unrestricted portfolio choice}

The optimization problem for an investor, with no constraints on the choice of optimal portfolio, can be formulated and solved by the martingale method of Cox and Huang. ${ }^{6}$ Recalling the dynamical completeness of the market an investor with a CRRA utility function solves the problem:

$$
\underset{\left\{W_{T}\right\}}{\operatorname{Max}} E_{0}\left[\frac{W_{T}^{1-\gamma}}{1-\gamma}\right]
$$

subject to the budget constraint

$$
W_{0}=E_{0}\left[M_{T} \cdot W_{T}\right] \quad[\mu]
$$

\footnotetext{
${ }^{5}$ This is also a consequence of the fact that the marginal utility of wealth tends to infinity as wealth tends to zero. For similar problems, where this is not the case, see e.g. chapter 6 in Merton (1992) and references cited in there.

${ }^{6}$ See Cox and Huang $(1989,1991)$ or Duffie (1996).
} 
The basic idea in this optimization approach is that wealth can be allocated in any way that is consistent with the budget constraint. And to the extent that preferences are only formulated for $W_{T}$ the only answer obtained in the first place is the optimal wealth distribution at time $T$. An explicit solution to the asset allocation decision at any point in time must be found afterwards by determining a dynamic trading strategy - along the lines well-known from contingent claims analysis - leading to the desired end-point distribution for $W_{T}$.

Theorem 1 (i) The optimal wealth distribution at the horizon $T$ is log-normally distributed and characterized by

$$
W_{T}=\frac{M_{T}^{-\frac{1}{\gamma}}}{E_{0}\left[M_{T}^{\Gamma}\right]} \cdot W_{0} \equiv W_{0} \cdot \exp \left[\mu_{W_{T}}-\frac{1}{2} \sigma_{W_{T}}^{2}-\sigma_{W_{T}} \mathcal{N}\right]
$$

where

- $\mathcal{N}$ is the same $N(0,1)$-variable as mentioned in (5)

- $\Gamma \equiv 1-\frac{1}{\gamma}$

- $\sigma_{W_{T}}=\frac{1}{\gamma} \sigma_{M_{T}}$

- $\mu_{W_{T}}=-\log (D(0 ; T))+\frac{1}{\gamma} \sigma_{M_{T}}^{2}=T y(0 ; T)+\frac{1}{\gamma} \sigma_{M_{T}}^{2}$

(ii) The optimal level of expected utility can - for $\gamma \neq 1$ - be written as

$$
J_{0}\left(W_{0} ; T, \gamma\right) \equiv E_{0}\left[\frac{W_{T}^{1-\gamma}}{1-\gamma}\right]=\frac{\left(\frac{W_{0}}{D(0 ; T)} \cdot \exp \left[\frac{1}{2 \gamma} \sigma_{M_{T}}^{2}\right]\right)^{1-\gamma}}{1-\gamma}
$$

For $\gamma=1$ this becomes

$$
J_{0}\left(W_{0} ; T, 1\right)=\log \left(W_{0}\right)+\frac{1}{2} \sigma_{M_{T}}^{2}-\log (D(0 ; T))
$$

(iii) The dynamic trading policy implementing the optimal wealth distribution depends upon the nature of the interest rate process.

- For a deterministic interest rate process the portfolio weights $\omega$ for risky assets is

$$
\omega=\frac{1}{\gamma} V\left(V^{\prime} V\right)^{-1} \lambda
$$

with the residual fraction of wealth $1-1_{N}^{\prime} \omega$ allocated to the risk-free asset.

- For a stochastic interest rate process the portfolio weights in (10) still apply. But the zero coupon bond expiring at time $T$ plays a special role as the risk-free asset relevant for the investment horizon. An additional fraction of wealth, $\Gamma$, is allocated into this particular bond or, equivalently, into a perfectly mimicking portfolio. The residual is allocated to the instantaneously risk-free asset. 
Proof (i) The first order condition for the optimization problem is given by the relation:

$$
W_{T}^{-\gamma}=\mu M_{T} \quad \Rightarrow \quad W_{T}=\left(\mu M_{T}\right)^{\left(-\frac{1}{\gamma}\right)}
$$

with the Lagrangian multiplier $\mu$ determined from the budget constraint:

$$
W_{0}=E_{0}\left[M_{T} W_{T}\right]=\mu^{-\frac{1}{\gamma}} M_{T}^{\Gamma} \Rightarrow \mu^{-\frac{1}{\gamma}}=\frac{W_{0}}{E_{0}\left[M_{T}^{\Gamma}\right]}
$$

Inserting this expression for $\mu^{-\frac{1}{\gamma}}$ in (11) proves the first equality in (7).

The fact that the pricing kernel $M_{T}$ is log-normally distributed, and can be represented in the functional form given in (5), implies that $M_{T}^{-\frac{1}{\gamma}}$ is also log-normally distributed:

$$
M_{T}^{-\frac{1}{\gamma}}=\exp \left\{-\frac{1}{\gamma}\left(\mu_{M_{T}}-\frac{1}{2} \sigma_{M_{T}}^{2}\right)-\frac{1}{\gamma} \sigma_{M_{T}} \mathcal{N}\right\}
$$

Hence $\sigma_{W_{T}}=(1 / \gamma) \sigma_{M_{T}}$.

The proofs of (ii), (iii), and the specific expression for $\mu_{W_{T}}$ require some tedious derivations, for which reason the details are devoted to Appendix A.

Corollary 1 Whenever there is no interest rate risk the expression for optimal expected utility simplifies for $\gamma \neq 1$ to

$$
J_{0}\left(W_{0} ; T, \gamma\right)=E_{0}\left[\frac{W_{T}^{1-\gamma}}{1-\gamma}\right]=\frac{\left(\frac{W_{0}}{D(0 ; T)} \cdot \exp \left[\frac{1}{2 \gamma}\|\lambda\|^{2} T\right]\right)^{1-\gamma}}{1-\gamma}
$$

For $\gamma=1$ the expression for optimal expected utility becomes

$$
J_{0}\left(W_{0} ; T, 1\right)=\log \left(W_{0}\right)+\left(r+\frac{1}{2}\|\lambda\|^{2}\right) T
$$

In the rest of the paper we will omit mentioning the special case $\gamma=1$. Most results are modified in an obvious manner.

\subsection{Optimal portfolio choice with interest rate guarantee}

Now assume that the investor is restricted in her final payoff profile by an exogenously given requirement that her final wealth must at least be her initial investment increased with a minimum guaranteed rate $g$, continuously compounded.

The investor's wealth at time $T$ under this restriction is denoted by $\widetilde{W}_{T}$, and the optimization problem is given as follows: 


$$
\underset{\left\{\widetilde{W}_{T}\right\}}{\operatorname{Max}} E_{0}\left[\frac{\widetilde{W}_{T}^{1-\gamma}}{1-\gamma}\right]
$$

subject to

$$
\begin{array}{ll}
W_{0}=E_{0}\left[M_{T} \widetilde{W}_{T}\right] & {\left[\mu_{0}\right]} \\
\widetilde{W}_{T} \geq W_{0} e^{g T} & {\left[\mu_{1}\right]}
\end{array}
$$

The first order conditions for this problem are given by:

$$
\widetilde{W}_{T}^{-\gamma}=\mu_{0} M_{T}-\mu_{1} \wedge \mu_{1} \cdot\left(\widetilde{W}_{T}-W_{0} e^{g T}\right)=0
$$

or, equivalently:

$$
\widetilde{W}_{T}= \begin{cases}\left(\mu_{0} M_{T}\right)^{\left(-\frac{1}{\gamma}\right)} & \text { if } \widetilde{W}_{T}>W_{0} e^{g T} \\ W_{0} e^{g T} & \text { otherwise }\end{cases}
$$

When $\mu_{1}>0$ the guarantee is effective and we have that $\widetilde{W}_{T}=W_{0} e^{g T}$. When $\mu_{1}=0$ the guarantee is not effective and we have that $\widetilde{W}_{T}=\left(\mu_{0} M_{T}\right)^{-1 / \gamma}$. I.e. whenever the guarantee is not effective the investor's payoff in the optimal solution $\widetilde{W}_{T}$ is proportional to $M_{T}^{-1 / \gamma}$, hence proportional to the payoff in the unrestricted case.

The factor of proportionality is called $x$ and is determined by the cost of insuring against "bad states", where the optimal wealth in the unrestricted case falls below the lower limit given by the guarantee.

Theorem 2 The optimal wealth distribution at the horizon $T, \widetilde{W}_{T}$, can be written as

$$
\begin{aligned}
\widetilde{W}_{T}=\max \left[W_{0} e^{g T}, x W_{T}\right] & =x W_{T}+\max \left[0, W_{0} e^{g T}-x W_{T}\right] \\
& =W_{0} e^{g T}+\max \left[0, x W_{T}-W_{0} e^{g T}\right]
\end{aligned}
$$

where $W_{T}$ is the solution found for the unconstrained problem and $x \in(0,1)$ is determined by the wealth constraint.

The optimal level of expected utility can be expressed as:

$$
\widetilde{J}_{0}\left(W_{0} ; T, \gamma\right)=U\left(W_{0} e^{g T}\right) N\left(d_{1}^{*}\right)+J_{0}\left(x W_{0} ; T, \gamma\right)\left(1-N\left(d_{2}^{*}\right)\right)
$$

where

$$
\begin{aligned}
d_{1}^{*} & =\frac{\gamma[(g-y(0 ; T)) T-\log (x)]}{\sigma_{M_{T}}}+\left(\frac{1}{2 \gamma}-1\right) \sigma_{M_{T}} \\
d_{2}^{*} & =d_{1}^{*}+\Gamma \sigma_{M_{T}} \\
& =\frac{\gamma[(g-y(0 ; T)) T-\log (x)]}{\sigma_{M_{T}}}-\frac{1}{2 \gamma} \sigma_{M_{T}}
\end{aligned}
$$


Proof The optimal wealth distribution is already proven in the derivation just before the statement of Theorem 2.

The optimal level of expected utility involves the following calculation:

$$
\begin{aligned}
\widetilde{J}_{0}\left(W_{0} ; T, \gamma\right) & =E_{0}\left[\frac{\left(\max \left[W_{0} e^{g T}, x W_{T}\right]\right)^{1-\gamma}}{1-\gamma}\right] \\
& =U\left(W_{0} e^{g T}\right) P\left\{W_{T} \leq K\right\}+E_{0}\left[\frac{\left(x W_{T}\right)^{1-\gamma}}{1-\gamma} \mathbf{1}_{\left\{W_{T}>K\right\}}\right]
\end{aligned}
$$

where

$$
K=\frac{W_{0} e^{g T}}{x}
$$

The rest is a standard calculation with truncated log-normally distributed variables. Details are given in Appendix B.

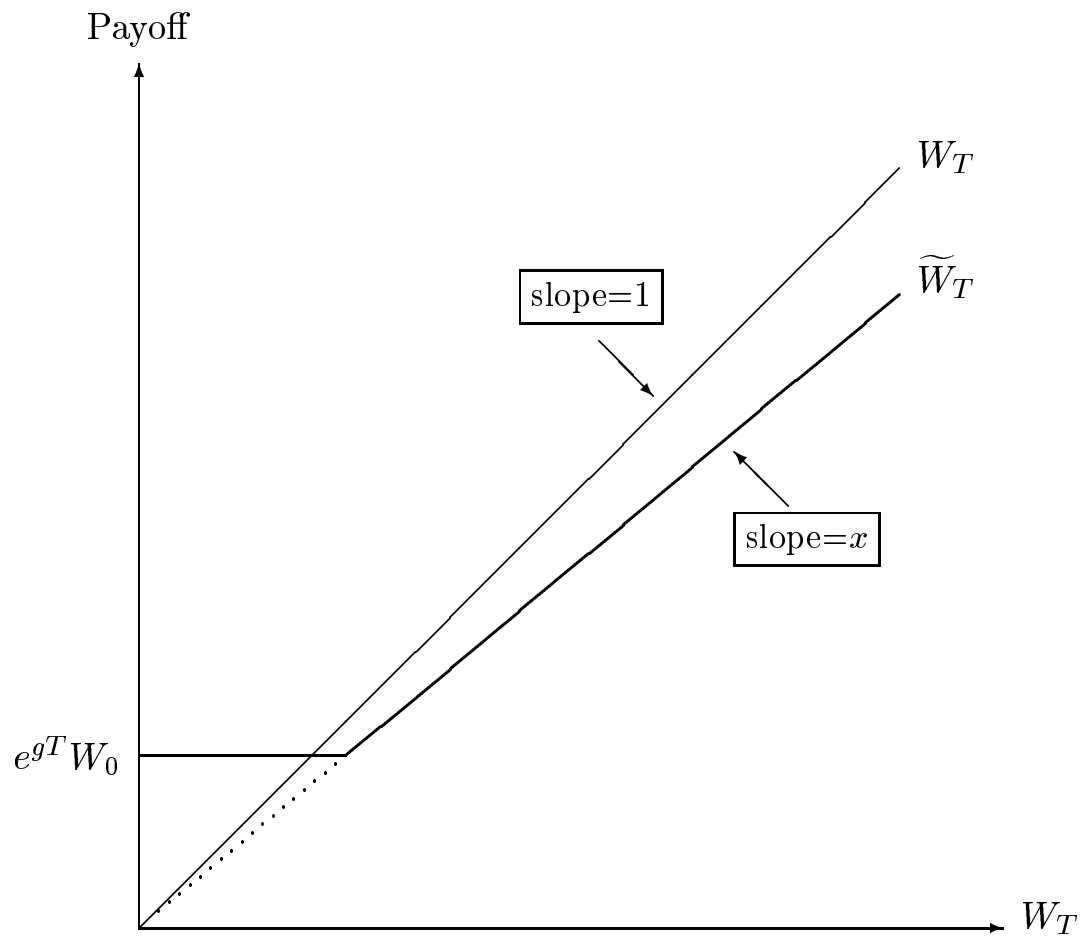

Figure 1: Payment profile as a result of a restricted $($ slope $=x)$ and an unrestricted (slope $=1$ ) optimal portfolio strategy.

The payoff profiles of the optimal portfolio strategies are shown in Figure 1. Observe that the put option involved can be duplicated by means of the assets making up the optimal unrestricted portfolio. Since duplicating a put option involves a short position in the underlying asset the 
effect of an interest rate guarantee is to limit the investment in the otherwise optimal risky portfolio.

The long term zero coupon bond matching the investment horizon $T$ becomes the risk-free asset relative to the investment horizon in question. Hence the long term zero coupon rate $y(0 ; T)$ matching the investment horizon $T$ becomes more interesting than the level of the short rate. The investment policy is driven towards $100 \%$ invested in this bond, when the guaranteed rate $g$ tends to $y(0 ; T)$.

The latter part within parenthesis on the rhs of (17) is equivalent to a put option with $x W_{T}$ as the underlying asset and with strike price $W_{0} e^{g T}$. The investor pays a fraction $1-x$ of her initial wealth for acquiring this put option. Denoting the price of this put as $P\left(W_{0}, g, T, x\right)$, we can deduce the following relations from the wealth constraint:

$$
\begin{aligned}
W_{0} & =x W_{0}+P\left(W_{0}, g, T, x\right) \Rightarrow \\
P\left(W_{0}, g, T, x\right) & =(1-x) W_{0}
\end{aligned}
$$

The price of the put option is known analytically, assuming the value of $x$ is known, in all the log-normal environments studied here.

Theorem 3 The put option price is given as

$$
P\left(W_{0}, g, T, x\right)=W_{0} \cdot\left[e^{g T} D(0 ; T) N\left(d_{1}^{*}+\sigma_{M_{T}}\right)-x N\left(d_{2}^{*}\right)\right]
$$

Proof The proof is a standard calculation with truncated log-normally distributed variables. The details are spelled out in Appendix B.

Plugging the put option price from (26) into the budget constraint (24) we have one equation with $x$ as the only unknown:

$$
1=x N\left(-d_{2}^{*}\right)+e^{(g-y(0 ; T)) T} N\left(d_{1}^{*}+\sigma_{M_{T}}\right)
$$

This equation is easily solved numerically for $x$.

\section{Wealth equivalents and comparative statics}

Our aim is to analyze the utility loss of imposing a minimum interest rate guarantee as an exogenous constraint on the investor's ability to tailor her portfolio. We define the wealth equivalent, denoted by $\widehat{W}_{0}$, as the amount of initial wealth necessary for the investor to achieve the same level of expected utility without restrictions imposed on the portfolio allocation as is possible with the initial wealth $W_{0}$ and the restriction imposed.

Since all relations are proportional in initial wealth we examine the wealth equivalent as a relative measure, i.e. as a fraction of the initial wealth maintained in utility terms despite the 
loss incurred upon the investor by enforcing the constraint. In the following we set $W_{0} \equiv 1$ without loss of generality.

We start by examining the comparative statics for $x$. We apply the implicit function theorem on (27) and use the symbol $F$ to define the rhs as a function of $x, g$ and $\gamma$. In doing so it is important to realize that

$$
e^{(g-y(0 ; T)) T} N^{\prime}\left(d_{1}^{*}+\sigma_{M_{T}}\right)=x N^{\prime}\left(-d_{2}^{*}\right)
$$

This relation implies that a number of terms cancel out in the expressions for partial derivatives, because the effect of any variable working through $d_{1}^{*}$ and $d_{2}^{*}$ with the same derivative effect on $d_{1}^{*}$ and $d_{2}^{*}$ cancels out. ${ }^{7}$ Hence

$$
\begin{aligned}
\frac{\partial F}{\partial x} & =N\left(-d_{2}^{*}\right)>0 \\
\frac{\partial F}{\partial g} & =T e^{(g-y(0 ; T)) T} N\left(d_{1}^{*}+\sigma_{M_{T}}\right)>0 \\
\frac{\partial F}{\partial \gamma} & =-x N^{\prime}\left(-d_{2}^{*}\right) \cdot \frac{\sigma_{M_{T}}}{\gamma^{2}}<0 \\
\frac{d x}{d g} & =-\frac{\partial F / \partial g}{\partial F / \partial x}=-\frac{T e^{(g-y(0 ; T)) T} N\left(d_{1}^{*}+\sigma_{M_{T}}\right)}{N\left(-d_{2}^{*}\right)}<0 \\
\frac{d x}{d \gamma} & =-\frac{\partial F / \partial \gamma}{\partial F / \partial x}=\frac{x N^{\prime}\left(-d_{2}^{*}\right)}{N\left(-d_{2}^{*}\right)} \cdot \frac{\sigma_{M_{T}}}{\gamma^{2}}>0
\end{aligned}
$$

The derivative $d x / d g$ is negative, implying that as the level of the interest rate guarantee increases, an increasing fraction of initial wealth must be allocated to the put option. Furthermore, it can be inferred from (27) that $\lim _{g \rightarrow y(0 ; T)} x=0$; i.e., when the interest rate guarantee is moving up and gets very close to the yield on the $T$-maturity zero coupon bond, the risk bearing capacity vanishes and the investment in the unrestricted optimal portfolio is eliminated. In this case the portfolio converges to a put option on an underlying asset $\left(x W_{T}\right)$ of zero value, equivalent to a portfolio position with $100 \%$ of zero coupon bonds with maturity date $T$. Note also that the convergence is such that $\lim _{g \rightarrow y(0 ; T)} d x / d g=-\infty$.

The derivative $d x / d \gamma$ is positive. As $\gamma$ changes, the optimal unrestricted portfolio leading to $W_{T}$ also changes. A very risk averse individual does not need the protection from the put option because the unrestricted wealth allocation already involves a high degree of built-in protection. This is also reflected in the fact that $\lim _{\gamma \rightarrow \infty} x=1$, as can be inferred from (27). ${ }^{8}$

Next we derive the comparative statics for the wealth equivalent. Finding the wealth equivalent amounts to solving the following equation, cf. (8) and (19):

$$
J_{0}\left(\widehat{W}_{0} ; T, \gamma\right)=\widetilde{J}_{0}(1 ; T, \gamma) \Leftrightarrow \widehat{W}_{0}=J_{0}^{-1} \circ \widetilde{J}_{0}(1 ; T, \gamma) \Leftrightarrow
$$

\footnotetext{
${ }^{7}$ This property is well-known from the Black-Scholes model and other option pricing formulas within the log-normal framework.

${ }^{8}$ Since we know from (33) that $x$ is an increasing function of $\gamma$, an asymptotic $\operatorname{limit}, \lim _{\gamma \rightarrow \infty} x$, exists in $(0,1]$. Then we also know that $d_{1}^{*}$ and $-d_{2}^{*}$ have opposite limiting infinite values. It is impossible that $\lim _{\gamma \rightarrow \infty} d_{1}^{*}=\infty$, because $e^{(g-y(0 ; T)) T}<1$. Hence $\lim _{\gamma \rightarrow \infty}\left(-d_{2}^{*}\right)=\infty$, which further implies that $\lim _{\gamma \rightarrow \infty} x=1$.
} 


$$
\begin{aligned}
\left(\frac{\widehat{W}_{0}}{D(0 ; T)} \exp \left(\frac{1}{2 \gamma} \sigma_{M_{T}}^{2}\right)\right)^{1-\gamma}= & \left(e^{g T}\right)^{1-\gamma} N\left(d_{1}^{*}\right)+ \\
& \left(\frac{x}{D(0 ; T)} \exp \left(\frac{1}{2 \gamma} \sigma_{M_{T}}^{2}\right)\right)^{1-\gamma}\left(1-N\left(d_{2}^{*}\right)\right)
\end{aligned}
$$

Equation (35) can be solved for analytically, once the variable $x$ has been determined. Using the chain rule and the definition of the wealth equivalent in (34) we arrive at

$$
\begin{aligned}
& \frac{d \widehat{W}_{0}}{d g}=\frac{\widehat{W}_{0}}{\begin{array}{c}
(1-\gamma) J_{0}\left(\widehat{W}_{0} ; T, \gamma\right) \\
+
\end{array}} \cdot\left[\begin{array}{cc}
\frac{\partial \widetilde{J}_{0}}{\partial g} & +\frac{\partial \widetilde{J}_{0}}{\partial x} \frac{\partial x}{\partial g} \\
+ & +
\end{array}\right] \\
& \frac{d \widehat{W}_{0}}{d \gamma}=\frac{\widehat{W}_{0}}{\begin{array}{c}
(1-\gamma) J_{0}\left(\widehat{W}_{0} ; T, \gamma\right) \\
+
\end{array}} \cdot[\underbrace{\frac{\partial \widetilde{J}_{0}}{\partial \gamma}-\frac{\partial J_{0}}{\partial \gamma}}_{?}+\frac{\partial \widetilde{J}_{0}}{\partial x} \frac{\partial x}{\partial \gamma}+\begin{array}{c}
+ \\
+
\end{array}]
\end{aligned}
$$

Analytical expressions for all the terms involving partial derivatives in (36) and (37) are found in Appendix C.

The expression in (36) reveals two effects of an increase in the level of the minimum interest rate guarantee $g$. The first term within the brackets is positive, reflecting the benefit from having a higher level of guaranteed payoff. The second term within the brackets is negative, reflecting the cost of paying for a higher level of guaranteed payoff. The second term must dominate the first term. The effect of increasing the level of $g$, as reflected in $d \widehat{W}_{0} / d g$, must be negative, because by increasing $g$ the set of feasible terminal payoffs of the restricted portfolio shrinks. A mathematical proof in the present context is found in Appendix C. Furthermore, $\lim _{g \rightarrow y(0 ; T)} d \widehat{W}_{0} / d g=-\infty$, which is also demonstrated in Appendix C.

The risk aversion parameter $\gamma$ enters through three channels:

- It changes the need for buying put options through a change in $x$ as a response to a change in $\gamma$.

- It changes the level of $\sigma_{W_{T}}$ in the unrestricted portfolio. This effect shows up in two places:

- The unrestricted expected utility changes.

- The cost of the put option changes.

- It changes the functional form of the utility function.

Numerical calculations for various parameter values suggest that $d \widehat{W}_{0} / d \gamma$ is positive but, due to the many effects of changing $\gamma$, we have not been able to prove this analytically in general neither have we been able to provide a counterexample.

Anyhow, a positive derivative $d \widehat{W}_{0} / d \gamma$ is consistent with the economic reasoning that, since $d x / d \gamma>0$, high values of $\gamma$ leads to a relatively high fraction of wealth invested in the unrestricted 
optimal portfolio. Hence, very risk averse investors do not suffer as much from the imposed constraints as do more risk tolerant investors. This effect is reflected in the latter positive term in (37).

\section{Examples}

Following the derivations in section 2 and section 3 above we observe that the indirect utility function and the wealth equivalent are solely determined by

- the discount factor $D(0 ; T)$ or, equivalently, the zero coupon rate $y(0 ; T)$

- the variance of the pricing kernel $\sigma_{M_{T}}^{2}$

- the relative risk aversion parameter $\gamma$

Whether the interest rate process is deterministic or stochastic has no direct influence on the results. Some of the calculations become more complex with a stochastic interest rate, but only because the calculation of $\sigma_{M_{T}}^{2}$ becomes more complex. Similarly, the zero coupon rate $y(0 ; T)$ - and not the spot rate $r_{0}$ - is the interest rate of primary concern. The distance between $y(0 ; T)$ and the level of the interest rate guarantee $g$ is gauging the severeness of the guarantee in the sense that the feasible set of investment opportunities shrinks as $g$ moves towards $y(0 ; T)$. When $g$ reaches the level $y(0 ; T)$ there is only one feasible investment policy: All wealth must be allocated to the discount bond expiring at time $T$.

In the following we consider two examples. Our primary example is the classical Black-Scholes framework, where there is only one stock (or portfolio of stocks), a constant risk premium, and a constant short term interest rate. Subsequently we consider the Black-Scholes model in combination with a Vasicek term structure model, cf. Vasicek (1977). In particular, we demonstrate how the parameter values can be chosen to obtain the same result for the wealth equivalent as in the classical Black-Scholes model.

\subsection{The classical Black-Scholes model}

The price process of the single risky asset (stock portfolio) is

$$
d S_{t}=\left(r+\lambda_{S} \sigma_{S}\right) S_{t} d t+\sigma_{S} S_{t} d Z_{t}=\mu_{S} S_{t} d t+\sigma_{S} S_{t} d Z_{t}
$$

Under this scenario the relations in Theorem 1 become $^{9}$

$$
\begin{aligned}
W_{t} & =W_{0} \cdot \exp \left[\left(r-\frac{1}{2} \frac{\lambda_{S}^{2}}{\gamma^{2}}+\frac{\lambda_{S}^{2}}{\gamma}\right) t+\frac{\lambda_{S}}{\gamma} Z_{t}\right] \\
W_{T} & =W_{0} \cdot \exp \left[\left(r-\frac{1}{2} \frac{\lambda_{S}^{2}}{\gamma^{2}}+\frac{\lambda_{S}^{2}}{\gamma}\right) T+\frac{\lambda_{S}}{\gamma} \sqrt{T} \mathcal{N}\right]
\end{aligned}
$$

\footnotetext{
${ }^{9}$ For details of the derivation see appendix A, in particular relation (67).
} 
where $\mathcal{N}$ is a $\mathrm{N}(0,1)$-variable. The level of expected utility is

$$
J_{0}\left(W_{0} ; T, \gamma\right)=W_{0}^{1-\gamma} \cdot \frac{\exp \left[(1-\gamma)\left(r+\frac{\lambda_{S}^{2}}{2 \gamma}\right) T\right]}{1-\gamma}
$$

and the dynamics of wealth follows the process:

$$
d W_{t}=W_{t}\left[\left(r+\frac{\lambda_{S}^{2}}{\gamma}\right) d t+\frac{\lambda_{S}}{\gamma} d Z_{t}\right]
$$

The optimal portfolio position in the risky asset that gives rise to this dynamics of wealth allocates a fixed fraction $\frac{\lambda_{S}}{\gamma \sigma_{S}}$ of wealth in the risky asset in accordance with Theorem 1 .

Besides the general comparative static results in section 3 it is obvious that we have the following limiting behaviour of the wealth equivalent:

$$
\begin{gathered}
\lim _{g \rightarrow-\infty} \widehat{W}_{0}=W_{0}, \quad \lim _{g \rightarrow-\infty} x=1 \\
\lim _{g \rightarrow r} \widehat{W}_{0}=W_{0} \exp \left(-\frac{\lambda_{S}^{2}}{2 \gamma} T\right), \quad \lim _{g \rightarrow r} x=0
\end{gathered}
$$

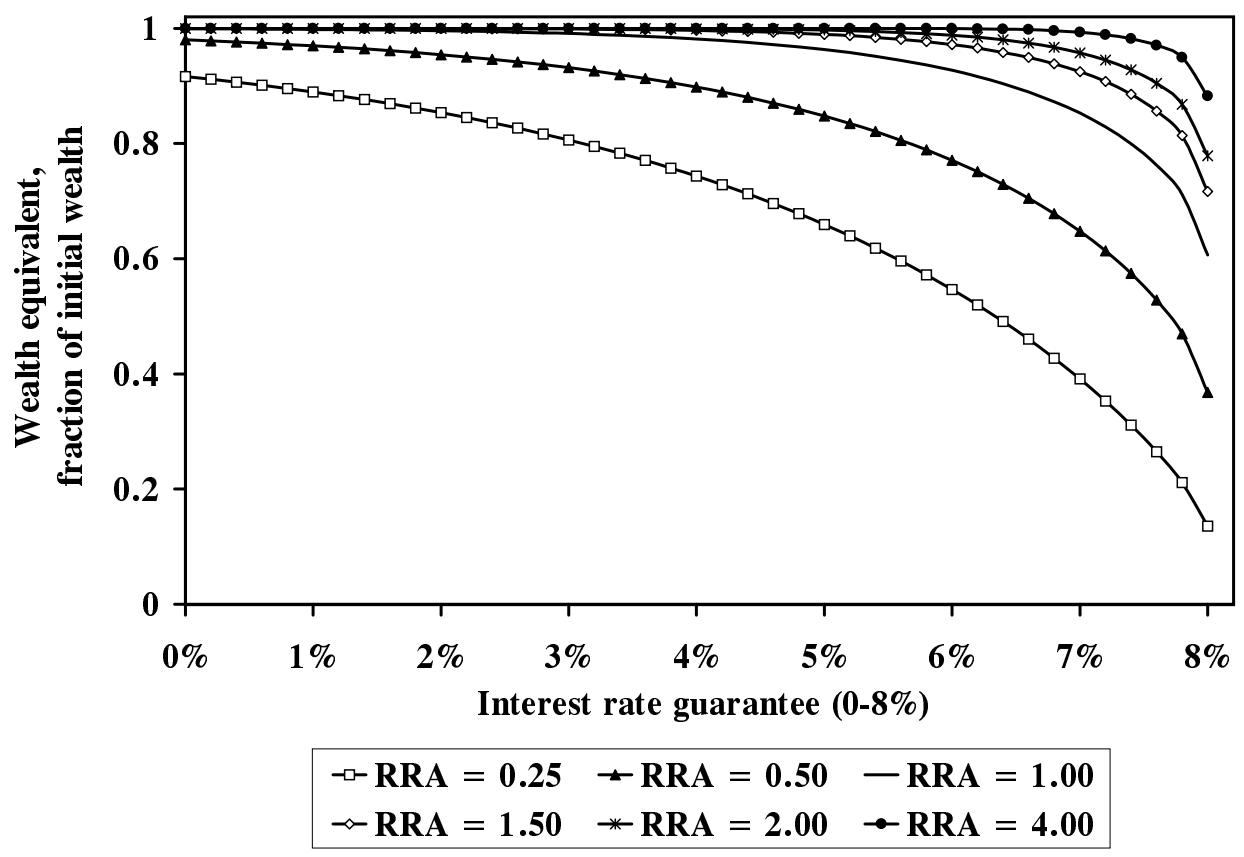

Figure 2: Wealth equivalents as a function of the interest rate guarantee for different investors. 
Figure 2 shows the wealth equivalents for varying levels of the interest rate guarantee between 0 and $8.0 \%$ and for a variety of values of the parameter $\gamma$, i.e. for a variety of values of the relative risk aversion (RRA). The following parameters are used:

$$
\mu_{S}=13 \%, \quad \sigma_{S}=25 \%, \quad r=8 \%, \quad \lambda_{S}=20 \%, \quad T=25
$$

The optimal unrestricted asset allocation is to invest the fraction $0.8 / \gamma$ in the stock portfolio. The logarithmic utility investor $(R R A=1)$ has $80 \%$ of wealth invested in stocks and $20 \%$ in risk-free assets in the unrestricted portfolio.

The shapes of the curves in Figure 2 are in accordance with the general comparative static results in section 3. As can be seen from Figure 2 the interest rate guarantee is turning into a severe constraint as the level of the guarantee approaches the level of the constant interest rate for all degrees of relative risk aversion, and this effect is increasing with decreasing level of risk aversion. The only way the minimum interest rate guarantee can be fulfilled is to switch the asset allocation much more heavily towards the bank account and away from the risky asset and its risk premium than would otherwise have been optimal.

For very low levels of the relative risk aversion $(\gamma \leq 0,5)$ even a zero guarantee is a perceptible restriction. In the other end, even a very risk averse investor with relative risk aversion 4 will suffer a measurable utility loss as the guaranteed rate $g$ moves towards the risk-free rate of interest $8 \%$.

\subsection{The Black-Scholes model with a Vasicek term structure}

As the second example we consider a menu of assets consisting of a stock portfolio, as in the classical Black-Scholes model, and a bond market driven by the Vasicek model.

The price processes can be written in accordance with the general notation outlined in (1). The Brownian motion $Z_{1 t}$ is chosen as the risk factor driving the stock investment opportunity, hence $\lambda_{1} \equiv \lambda_{S}$. The Brownian motion $Z_{2 t}$ is chosen as independent of $Z_{1 t}$. The correlation coefficient between the return processes in these two markets is denoted by $\rho$ and is assumed constant.

With our choice of specification, the Vasicek model is given by the following set of stochastic differential equations for the stock price process $\left(S_{t}\right)$, the price process for a zero coupon bond with maturity $H(D(t ; H))$ and the process for the infinitesimally risk-free rate of interest $\left(r_{t}\right)$. The coefficients are adjusted in order to get the variance of the bond price processes as well as the correlation $\rho$ correct:

$$
\begin{aligned}
{\left[\begin{array}{c}
d S_{t} / S_{t} \\
d D(t ; H) / D(t ; H) \\
d r_{t}
\end{array}\right]=} & {\left[\begin{array}{c}
r_{t}+\lambda_{S} \sigma_{S} \\
r_{t}+\lambda_{r} \sigma_{r} B(H-t) \\
a\left(b-r_{t}\right)
\end{array}\right] d t+} \\
& {\left[\begin{array}{cc}
\sigma_{S} & 0 \\
\rho \sigma_{r} B(H-t) & \sigma_{r} \sqrt{1-\rho^{2}} B(H-t) \\
-\rho \lambda_{S} \sigma_{r} & -\lambda_{2} \sigma_{r} \sqrt{1-\rho^{2}}
\end{array}\right]\left[\begin{array}{c}
d Z_{1 t} \\
d Z_{2 t}
\end{array}\right] }
\end{aligned}
$$

where

$$
\lambda_{r} \equiv \lambda_{S} \rho+\lambda_{2} \sqrt{1-\rho^{2}}
$$


It is well-known that the bond prices have the following form:

$$
\begin{aligned}
D(t ; H) & =\exp \left[A(H-t)-B(H-t) r_{t}\right] \\
A(\tau) & =r_{\infty}(B(\tau)-\tau)-\frac{\sigma_{r}^{2} B(\tau)^{2}}{4 a} \\
r_{\infty} & =b+\frac{\lambda_{r} \sigma_{r}}{a}-\frac{\sigma_{r}^{2}}{2 a^{2}} \\
B(\tau) & =\frac{1-e^{-a \tau}}{a}
\end{aligned}
$$

As shown in Theorem 1 the unrestricted portfolio policy in this case is a combination of

- a fraction $\Gamma$ invested in the zero coupon bond expiring at time $T$,

- a "speculative portfolio" with the portfolio weights given in (10),

- and the residual allocated to the instantaneously risk-free asset.

The volatility of the pricing kernel, $\sigma_{M_{T}}^{2}$, involves some straightforward calculations with integrals of $B(T-s)$ and $B(T-s)^{2}$. It has the form

$$
\begin{aligned}
\sigma_{M_{T}}^{2} & =\int_{0}^{T}\left(\left[\lambda_{S}-\rho \sigma_{r} B(T-s)\right]^{2}+\left[\lambda_{2}-\sigma_{r} \sqrt{1-\rho^{2}} B(T-s)\right]^{2}\right) d s \\
& =\left(\lambda_{S}^{2}+\lambda_{2}^{2}\right) T+2\left(r_{\infty}-b\right)(B(T)-T)-\frac{\sigma_{r}^{2}}{2 a} B(T)^{2}
\end{aligned}
$$

By choosing parameters that give rise to the same magnitude of $\sigma_{M_{T}}^{2}$ and $D(0 ; T)$ as in the former example with the classical Black-Scholes model, the wealth equivalents will be exactly the same as displayed in Figure 2. In the classical Black-Scholes model $\sigma_{M_{T}}^{2}=1$ and $D(0 ; T)=e^{-2}$. This is accomplished by, e.g., choosing parameter values

$$
\begin{gathered}
a=0.2, \quad b=0.08, \quad \lambda_{r}=0.05, \quad \sigma_{r}=0.0228, \quad \lambda_{2}=0 \\
\lambda_{S}=0.2, \quad \sigma_{S}=0.25, \quad \rho=0.25, \quad T=25
\end{gathered}
$$

With these parameter values the risk-premium at time 0 on the 25 year zero coupon bond is close to $0.57 \%$, whereas the risk premium on the stock portfolio is $5 \%$. The parameters above for the interest rate process are close to the estimates for the US market found in Chan et al. (1992) for the Vasicek model. The positive correlation between the returns on stocks and bonds is suggested by, e.g., Campbell (1987), Fama and French (1989) and Shiller and Beltratti (1992).

Note that the magnitude of $\sigma_{S}$ has no role to play for $\sigma_{M_{T}}$ and $D(0 ; T)$, but it is crucial for the exact portfolio composition implementing the optimal portfolio policy. With the parameters above the optimal portfolio for a logarithmic utility investor, cf. Theorem 1, $80 \%$ is in the stock portfolio and $20 \%$ in the bank account. For an investor with relative risk aversion $\gamma=2$ the investor allocates $40 \%$ to the stock portfolio, $50 \%$ to the zero coupon bond expiring at the horizon and the residual $10 \%$ in the bank account. 


\section{$5 \quad$ Heterogenous investors}

When an individual is a member of an investment pool the sharing of the realized portfolio value is assumed to be linear on a pro rata basis. This is the usual restriction forced upon the investor's payoff profile in pension schemes, participating life insurance contracts and other institutional savings arrangements.

There are only few results in the finance literature for this situation to be Pareto optimal. For CRRA utility functions this requires identical degrees of relative risk aversion, in which case the investment objective for the fund manager of the pool is obvious.

The interesting questions arise when the members of the pool have CRRA utility functions with different degrees of relative risk aversion - or even very different utility functions. In this case it is well known that non-linear sharing rules are necessary in order for the members of the pool to share the portfolio value in a Pareto optimal way. However, we take the pro rata sharing as a given institutional restriction and consider an investor with a CRRA utility function who participates in a fund where the investment policy is deviating from her own preferred unrestricted optimal investment policy.

As an analytically convenient behavioral rule for the fund manager we assume in this section that the fund manager invests in the same manner as would an investor with some CRRA utility function with a relative risk aversion parameter $\bar{\gamma}$ different from that of the individual investor under consideration. This behavioral rule could be loosely interpreted as an attempt on behalf of the fund manager to compromise between individual preferences.

We have the following result for the value of the expected utility of a CRRA investor.

Theorem 4 (i) If wealth is invested according to what is optimal for investors with investment horizon $T$ and constant risk aversion parameter $\bar{\gamma}$, then the level of expected utility of an investor with a CRRA utility function from wealth at the investment horizon $T$ and constant relative risk aversion parameter $\gamma$ can be expressed as:

$$
J_{0}\left(W_{0} ; T \gamma \mid \bar{\gamma}\right)=\frac{\left(\frac{W_{0}}{D(0 ; T)} \cdot \exp \left[\frac{1}{2 \gamma}\left(1-\left(\frac{\bar{\gamma}-\gamma}{\bar{\gamma}}\right)^{2}\right) \sigma_{M_{T}}^{2}\right]\right)^{1-\gamma}}{1-\gamma}
$$

(ii) If wealth is invested with an interest rate guarantee and according to what is constrained optimal for investors with investment horizon $T$ and constant risk aversion parameter $\bar{\gamma}$ as described in Theorem 2, then the level of expected utility of an investor with CRRA utility function $U(\cdot)$ from wealth at the investment horizon $T$ and constant relative risk aversion parameter $\gamma$ can be expressed as:

$$
\widetilde{J}_{0}\left(W_{0} ; T, \gamma \mid \bar{\gamma}\right)=U\left(W_{0} e^{g T}\right) N\left(\bar{d}_{1}\right)+J_{0}\left(x W_{0} ; T, \gamma \mid \bar{\gamma}\right)\left(1-N\left(\bar{d}_{2}\right)\right)
$$

with

$$
\begin{aligned}
& \bar{d}_{1}=\frac{\bar{\gamma}[(g-y(0 ; T)) T-\log (x)]}{\sigma_{M_{T}}}+\left(\frac{1}{2 \bar{\gamma}}-1\right) \sigma_{M_{T}} \\
& \bar{d}_{2}=\bar{d}_{1}+\frac{\gamma-1}{\bar{\gamma}} \sigma_{M_{T}}
\end{aligned}
$$


and where $x$ is determined so that the budget constraint is satisfied with equality, as described in Theorem 2.

Proof The proof of (i) follows from the log-normality of $W_{T}$ and is a straightforward extension of the proof of Theorem 1, part (ii), as given in Appendix A. Likewise the proof of (ii) is a straightforward extension of the proof of the form of the indirect utility function in Theorem 2 as given in Appendix B.

Corollary 2 For an unrestricted investment policy the wealth equivalent $\widehat{W}_{0}$ is given by

$$
\widehat{W}_{0}=W_{0} \cdot \exp \left[-\frac{1}{2 \gamma}\left(1-\frac{\gamma}{\bar{\gamma}}\right)^{2} \sigma_{M_{T}}^{2}\right]
$$

Proof Follows directly from equation (50) in combination with equation (8).

In Figure 3 we show the relation (52) with $W_{0}=1$ for three different values of the relative risk aversion parameter $\bar{\gamma}$ applied in the pool: $0.5,1$ and 2. We have applied the same parameter values as used in section 4.1. As is apparent from Figure 3 forced participation in an investment pool on a pro rata basis can induce severe utility losses, especially for relatively risk tolerant investors.

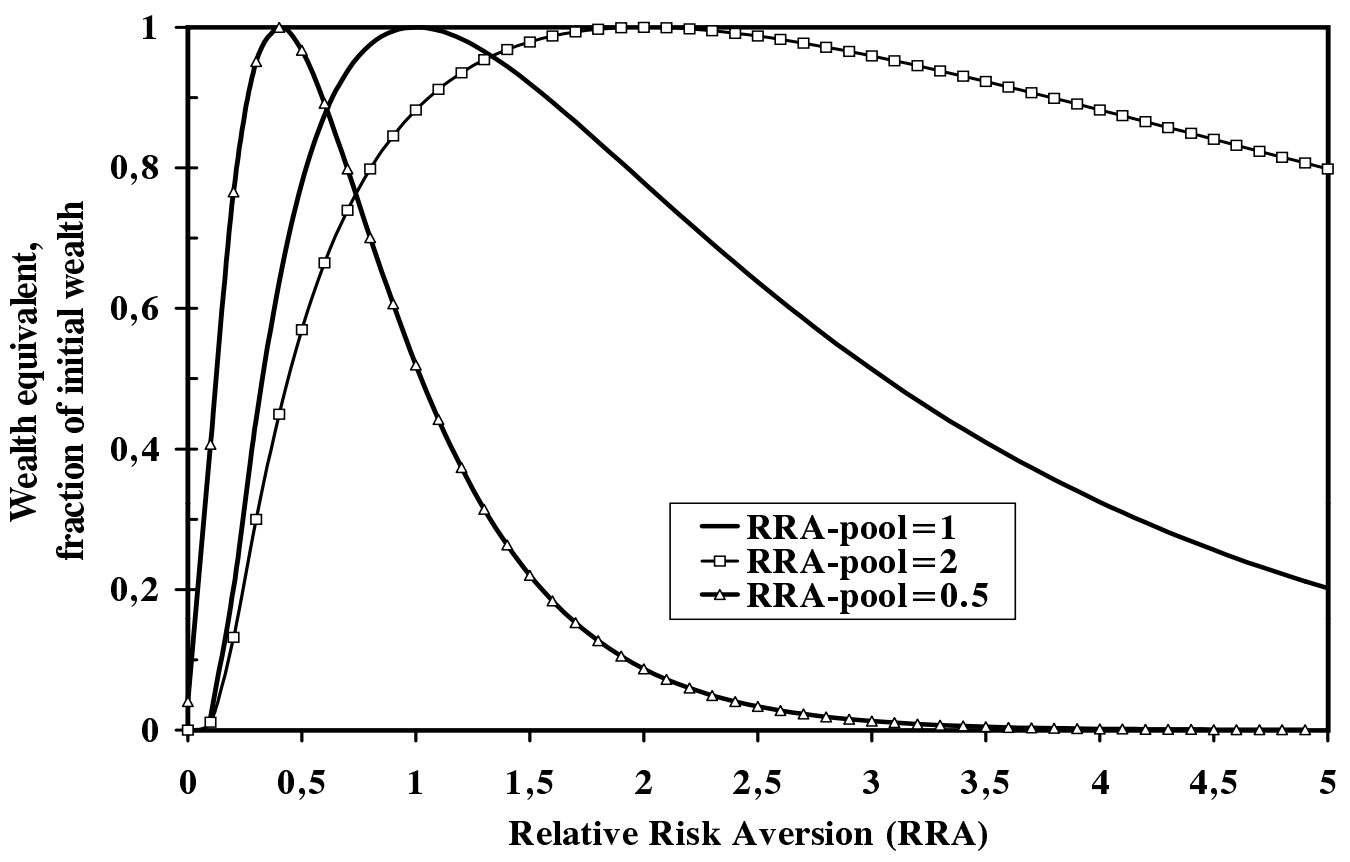

Figure 3: Wealth equivalents as a function of individual relative risk aversion (RRA) for investors participating in an investment pool with portfolio policies determined by $\bar{\gamma}=0.5, \bar{\gamma}=1$, and $\bar{\gamma}=2$, respectively. 
When investors in a pool are also subject to a minimum interest rate guarantee the situation is no longer clear-cut. Everyone suffers a loss of utility because of the compromise between preferences. However, the minimum interest rate guarantee is a compensation for some investors for a too "aggressive" investment policy, whereas other investors suffer an additional utility loss due to a too "conservative" investment policy.

Figure 4 shows this within the framework of the classical Black-Scholes model and with the same parameter values as in section 4.1. The figure shows the wealth equivalent as a function of the interest rate guarantee $g$ for different CRRA investors participating in a pool, where the investment policy in the pool is in accordance with the preferences of a logarithmic investor, i.e. $\bar{\gamma}=1$. The asymptotic values "to the left", $\lim _{g \rightarrow-\infty} \widehat{W}_{0}$, are identical to the wealth equivalent in (52).

For the logarithmic investor the curve is identical to the analogous curve in Figure 2. For investors with a relative risk aversion higher than the logarithmic investor, $\gamma>1$, the wealth equivalent is increasing as a function of the minimum interest guarantee $g$ until $g$ gets close to the level of the risk-free rate of interest. For investors with a relative risk aversion lower than the logarithmic investor, $\gamma<1$, the wealth equivalent is decreasing as a function of the minimum interest guarantee $g$ everywhere.

In all cases the very last segments of the curves in Figure 4 are coinciding with the analogous curves in Figure 2. When $g$ gets close to the level of the risk-free rate of interest the feasible set of investment policies shrinks and becomes almost independent of the compromised preferences in the pool.

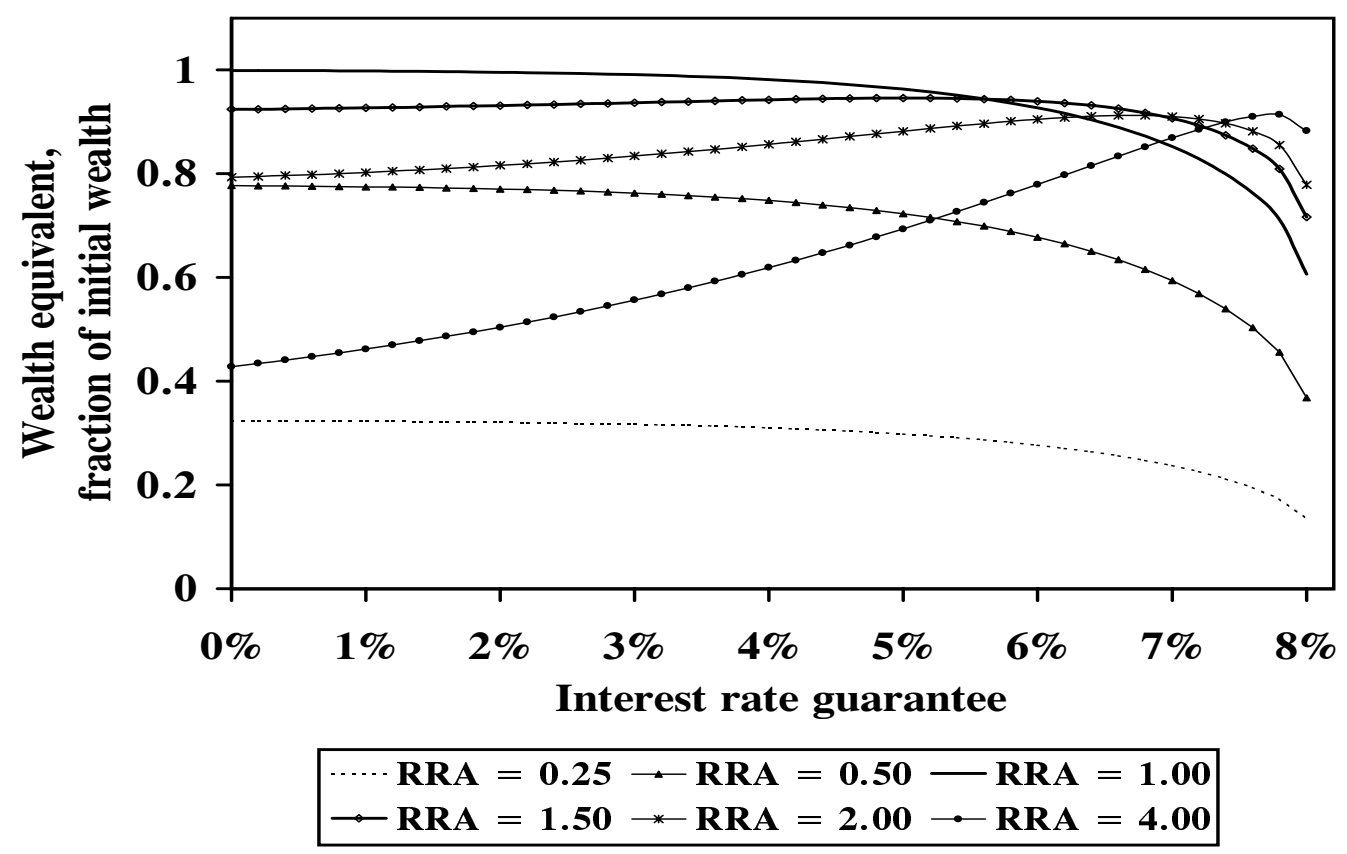

Figure 4: Wealth equivalents as a function of the interest rate guarantee $g$ for different investors participating in an investment pool with portfolio policy determined by $\bar{\gamma}=1$. 


\section{Conclusion and topics for further investigation}

The common feature of minimum interest rate guarantees in defined contribution pension schemes may be viewed as an exogenously imposed constraint on individual portfolio optimization that cannot easily be offset by the individual. In this paper we have demonstrated that such an exogenously imposed restriction may cause severe utility losses. This is different from the conventional view that interest rate guarantees are useful embedded options that the investor would find it fair to pay for. In fact the investor is paying for this embedded option through the asset allocation decisions, but should be reluctant to do so whenever the embedded option is not part of her unrestricted optimal investment policy.

The utility loss due to the interest rate guarantee is most outspoken for relatively risk tolerant investors. Furthermore, when investors participate in an investment pool on a pro rata basis the interest rate guarantee tends to reinforce this utility loss. On the other hand, for relatively risk averse investors in an investment pool an interest rate guarantee tends to work as a defensive mechanism towards a too risk tolerant investment policy in the pool.

An interesting topic for future research in the context of a minimum interest rate guarantee is to examine the effects of annual guarantees as also found in many real world contracts. This will clearly reinforce the effects found here. Also, the effects of an interest rate guarantee in a pool of individuals, heterogenous w.r.t. their age profile, seems an interesting topic for further investigation. 


\section{References}

Aase, K. AND S.-A. Persson (1997): "Valuation of the minimum guaranteed return embedded in life insurance products," Journal of Risk and Insurance, Forthcoming.

Albizzati, M. O. And H. Geman (1994): "Interest rate risk management and valuation of the surrender option in life insurance policies," Journal of Risk and Insurance, 62:616-637.

Ang, A. AND G. BeKaERT (1999): "International asset allocation with time-varying correlations," Working Paper, Graduate School of Business, Stanford University.

Bacinello, A. AND F. ORTU (1993a): "Pricing equity-linked life insurance with endogenous guarantees," Insurance: Mathematics and Economics, 12:245-257.

- (1993b): "Pricing guaranteed securities-linked life insurance under interest-rate risk," Actuarial Approach for Financial Economics, 3:195-213.

BASAK, S. (1995): “A general equilibrium model of portfolio insurance," The Review of Financial Studies, 8(4):1059-1090.

Brennan, M. J. AND E. S. Schwartz (1976): "The pricing of equity-linked life insurance policies with an asset value guarantee," Journal of Financial Economics, 3:195-213.

(1979): "Alternative investment strategies for the issuers of equity-linked life insurance policies," Journal of Business, 52:63-93.

Brennan, M. J., E. S. Schwartz, and R. Lagnado (1997): "Strategic asset allocation," Journal of Economic Dynamics and Control, 21(8-9):1377-1403.

Campbell, J. Y. (1987): "Stock returns and the term structure," Journal of Financial Economics, 18:373-399.

Campbell, J. Y. And L. Viceira (1998): "Who should buy long term bonds," NBER Working paper, No. 6801.

(1999): "Consumption and portfolio decisions when expected returns are time varying," The Quarterly Journal of Economics, 114:433-495.

Chan, K. C., G. A. Karolyi, F. A. Longstaff, and A. B. Sanders (1992): "The volatility of short-term interest rates: An empirical comparison of alternative models of the short rate," The Journal of Finance, 47(3):1209-1227.

Cox, J. C. AND C.-F. HuAng (1989): "Optimal consumption and portfolio policies when asset prices follow a diffusion proces," Journal of Economic Theory, 49:33-83.

Cox, J. C. AND C.-F. HuAng (1991): “A variational problem arising in financial economics," Journal of Mathematical Economics, 20:465-487.

DAS, S. R. AND R. UPPAL (1996): "International portfolio choice with stochastic correlations," Working paper. 
Duffie, D. (1996): Dynamic Asset Pricing Theory, Princeton University Press, Princeton, New Jersey, USA. 2nd edition.

FAMA, E. F. AND K. R. FRENCH (1989): "Business conditions and expected returns on stocks and bonds," Journal of Financial Economics, 25:23-49.

Grosen, A. And P. L. Jørgensen (1997): "Valuation of early exercisable interest rate guarantees," Journal of Risk and Insurance, 64:481-503.

(1999): "Fair valuation of life insurance liabilities: The impact of interest rate guarantees, surrender options and bonus policies," Working paper, Department of Management, University of Aarhus. Forthcoming in Insurance: Mathematics and Economics.

Grossman, S. J. AND Z. ZHOU (1996): "Equilibrium analysis of portfolio insurance," The Journal of Finance, 51:1379-1403.

Huang, C.-F. And R. H. Litzenberger (1988): Foundations for Financial Economics, North-Holland, New York, USA.

Merton, R. C. (1971): "Optimum consumption and portfolio rules in a continuous time model," Journal of Economic Theory, 3:373-413. Erratum: Merton (1973). Reprinted in Merton (1992, Chapter 5).

(1973): "Erratum," Journal of Economic Theory, 6:213-214.

(1992): Continuous-Time Finance, Basil Blackwell Inc., Cambridge, Massachusetts.

NiElsen, J. And K. SAndmann (1995): "Equity-linked life insurance - A model with stochastic interest rates," Insurance: Mathematics and Economics, 16:225-253.

(1996): "Uniqueness of the fair premium for equity-linked life insurance contracts," Geneva Papers on Risk and Insurance Theory, 21:65-102.

Rubinstein, M. (1976): "The valuation of uncertain income streams and the pricing of options," The Bell Journal of Economics, 7:407-425.

(1985): "Alternative paths to portfolio insurance," Financial Analysts Journal, $41(4): 42-52$.

Shiller, R. J. AND A. E. Beltratti (1992): "Stock prices and bond yields: Can their comovements be explained in terms of present value models?," Journal of Monetary Economics, $30: 25-46$.

Sørensen, C. (1999): "Dynamic asset allocation and fixed income management," Journal of Financial and Quantitative Analysis, 34(4):513-531.

VASICEK, O. (1977): "An equilibrium characterization of the term structure," Journal of Financial Economics, 5:177-188. 


\section{Appendix}

\section{A Theorem 1 (Continuation of proof)}

(ii) In order to find the expression for the optimal expected utility we elaborate on the following expression, based on the first equality in (7)

$$
W_{T}^{1-\gamma}=\frac{M_{T}^{\Gamma}}{\left(E_{0}\left[M_{T}^{\Gamma}\right]\right)^{1-\gamma}} \cdot W_{0}^{1-\gamma} \Rightarrow E_{0}\left[W_{T}^{1-\gamma}\right]=\left(E_{0}\left[M_{T}^{\Gamma}\right]\right)^{\gamma} \cdot W_{0}^{1-\gamma}
$$

First we find $\left(E_{0}\left[M_{T}^{\Gamma}\right]\right)^{\gamma}$. From the log-normality of $M_{T}$ :

$$
M_{T}=\exp \left\{\mu_{M_{T}}-\frac{1}{2} \sigma_{M_{T}}^{2}+\sigma_{M_{T}} \mathcal{N}\right\}
$$

it follows that $M_{T}^{\Gamma}$ is log-normal as well:

$$
M_{T}^{\Gamma}=\exp \left\{\Gamma\left(\mu_{M_{T}}-\frac{1}{2} \sigma_{M_{T}}^{2}\right)+\Gamma \sigma_{M_{T}} \mathcal{N}\right\}
$$

Hence,

$$
\begin{aligned}
E_{0}\left[M_{T}^{\Gamma}\right] & =\exp \left[\Gamma \mu_{M_{T}}-\frac{1}{2 \gamma} \Gamma \sigma_{M_{T}}^{2}\right] \Rightarrow \\
\left(E_{0}\left[M_{T}^{\Gamma}\right]\right)^{\gamma} & =\exp \left[(1-\gamma)\left(-\mu_{M_{T}}\right)+\frac{1-\gamma}{2 \gamma} \sigma_{M_{T}}^{2}\right] \\
& =\exp \left[-\mu_{M_{T}}+\frac{1-\gamma}{2 \gamma} \sigma_{M_{T}}^{2}\right]^{1-\gamma}
\end{aligned}
$$

Recall that

$$
D(0 ; T)=\exp \left(\mu_{M_{T}}\right) \quad \Leftrightarrow \quad-\log (D(0 ; T))=-\mu_{M_{T}}
$$

Insert (56) in the expression for $W_{T}$ in (7) to obtain the expression for the parameter $\mu_{W_{T}}$. This concludes the proof of (i).

Next insert (58) into (57) and (57) into (53) to obtain

$$
E_{0}\left[W_{T}^{1-\gamma}\right]=\left(\frac{W_{0}}{D(0 ; T)} \cdot \exp \left[\frac{1}{2 \gamma} \sigma_{M_{T}}^{2}\right]\right)^{1-\gamma}
$$

This immediately gives the expression for $J\left(W_{0}, T ; \gamma\right)$ in $(8)$. For the logarithmic case, i.e. $\gamma=1$, we have that

$$
\begin{aligned}
\log \left(W_{T}\right) & =\log \left(W_{0}\right)-\log \left(M_{T}\right)=\log \left(W_{0}\right)+\left(\frac{1}{2} \sigma_{M_{T}}^{2}-\mu_{M_{T}}+\sigma_{M_{T}} \mathcal{N}\right) \quad \Rightarrow \\
E_{0}\left[\log \left(W_{T}\right)\right] & =\log \left(W_{0}\right)+\left(\frac{1}{2} \sigma_{M_{T}}^{2}-\log (D(0 ; T))\right.
\end{aligned}
$$


which is the expression for $J\left(W_{0}, T ; 1\right)$ in $(9)$.

(iii) Resorting to the martingale property of $M_{t} W_{t}$ we have that

$$
\begin{aligned}
M_{t} W_{t} & =E_{t}\left[\frac{M_{T}^{\Gamma}}{E_{0}\left[M_{T}^{\Gamma}\right]}\right] \cdot W_{0} \quad \Rightarrow \\
W_{t} & =M_{t}^{-1} \cdot E_{t}\left[\frac{M_{T}^{\Gamma}}{E_{0}\left[M_{T}^{\Gamma}\right]}\right] \cdot W_{0} \equiv W_{0} \cdot M_{t}^{-1} \cdot \Psi_{t}
\end{aligned}
$$

where

$$
\Psi_{t}=E_{t}\left[\frac{M_{T}^{\Gamma}}{E_{0}\left[M_{T}^{\Gamma}\right]}\right]
$$

is a martingale gauging the deviation from the optimal growth portfolio, $W_{0} M_{t}^{-1}$, which describes the optimal wealth process for the special case of a logarithmic utility investor $(\gamma=1)$.

From (55) and (57) we have that

$$
\Psi_{T}=\frac{M_{T}^{\Gamma}}{E_{0}\left[M_{T}^{\Gamma}\right]}=\exp \left[\Gamma \sigma_{M_{T}} \mathcal{N}-\frac{1}{2} \Gamma^{2} \sigma_{M_{T}}^{2}\right]
$$

Under deterministically evolving interest rates the optimal portfolio policy and the dynamics of optimal wealth simplifies considerably. Using the characterization of $M_{t}$ in (4) and redoing the calculations in (55)-(57), we obtain

$$
\begin{aligned}
\frac{M_{T}^{\Gamma}}{E_{0}\left[M_{T}^{\Gamma}\right]} & =\exp \left[-\Gamma \lambda^{\prime} Z_{T}-\frac{1}{2} \Gamma^{2}\|\lambda\|^{2} T\right] \Rightarrow \\
\Psi_{t} & =E_{t}\left[\exp \left[-\Gamma \lambda^{\prime} Z_{T}-\frac{1}{2} \Gamma^{2}\|\lambda\|^{2} T\right]\right]=\exp \left[-\Gamma \lambda^{\prime} Z_{t}-\frac{1}{2} \Gamma^{2}\|\lambda\|^{2} t\right]
\end{aligned}
$$

The optimal unrestricted portfolio in (62) under deterministically evolving interest rates can now be stated in a compact manner:

$$
\begin{aligned}
W_{t} & =W_{0} \cdot M_{t}^{-1} \cdot \Psi_{t} \\
& =W_{0} \cdot \exp \left[h_{t}+(1-\Gamma) \lambda^{\prime} Z_{t}+\frac{1}{2}\left(1-\Gamma^{2}\right)\|\lambda\|^{2} t\right] \\
& =W_{0} \cdot \exp \left[h_{t}+\left(\frac{1}{\gamma}-\frac{1}{2 \gamma^{2}}\right)\|\lambda\|^{2} t+\frac{1}{\gamma} \lambda^{\prime} Z_{t}\right]
\end{aligned}
$$

with the dynamics

$$
d W_{t}=W_{t} \cdot\left[\left(r_{t}+\frac{\|\lambda\|^{2}}{\gamma}\right) d t+\frac{1}{\gamma} \lambda^{\prime} d Z_{t}\right]
$$

The portfolio policy is then found in a straightforward manner from combining the price dynamics (1) with the development in the optimal portfolio in (68). As stated in (10) a solution can be found by applying the generalized inverse:

$$
\omega=\frac{1}{\gamma} V\left(V^{\prime} V\right)^{-1} \lambda
$$


in accordance with the classical results of Merton (1971). In the case where $N=M$ this solution is unique. In the case where $N>M$ this solution is not unique, but one among an infinity of possible solutions for the optimal portfolio.

Under interest rate uncertainty things become slightly more complex. The relevant pricing kernel at any intermediate point in time, $t$, is $M_{T} / M_{t}$ :

$$
\begin{aligned}
& M_{T} / M_{t}=\exp \left[-\left(h_{T}-h_{t}\right)-\lambda^{\prime}\left(Z_{T}-Z_{t}\right)-\frac{1}{2}\|\lambda\|^{2}(T-t)\right] \\
& =\exp \left[h_{t}-\bar{h}_{T \mid t}-\frac{1}{2}\|\lambda\|^{2}(T-t)-\left(h_{T}-\bar{h}_{T \mid t}+\lambda\left(Z_{T}-Z_{t}\right)\right)\right] \\
& \equiv \exp \left[\mu_{M_{T \mid t}}-\frac{1}{2} \sigma_{M_{T \mid t}}^{2}+\sigma_{M_{T \mid t}} \mathcal{N}\right] \\
& \bar{h}_{T \mid t}=E_{t}\left[h_{T}\right] \\
& \sigma_{M_{T \mid t}}^{2}=\operatorname{var}_{t}\left(\log \left(M_{T}\right)-\log \left(M_{t}\right)\right)=\operatorname{var}_{t}\left(h_{T}-h_{t}+\lambda^{\prime}\left(Z_{T}-Z_{t}\right)\right) \\
& \mu_{M_{T \mid t}}=h_{t}-\bar{h}_{T \mid t}-\frac{1}{2}\|\lambda\|^{2}(T-t)+\frac{1}{2} \sigma_{M_{T \mid t}}^{2} \\
& D(t ; T)=E_{0}\left[\left(M_{T} / M_{t}\right)\right] \equiv \exp \left(\mu_{M_{T \mid t}}\right) \\
& M_{T}^{\Gamma}=M_{t}^{\Gamma} \cdot\left(M_{T} / M_{t}\right)^{\Gamma} \\
& =M_{t}^{\Gamma} \cdot D(t ; T)^{\Gamma} \cdot \exp \left[\Gamma\left(\sigma_{M_{T \mid t}} \mathcal{N}-\frac{1}{2} \sigma_{M_{T \mid t}}^{2}\right)\right] \\
& E_{t}\left[M_{T}^{\Gamma}\right]=M_{t}^{\Gamma} \cdot D(t ; T)^{\Gamma} \cdot \exp \left[-\frac{1}{2 \gamma} \Gamma \sigma_{M_{T \mid t}}^{2}\right] \\
& \Psi_{t}=M_{t}^{\Gamma} \cdot\left(\frac{D(t ; T)}{D(0 ; T)}\right)^{\Gamma} \cdot \exp \left[\frac{1}{2 \gamma} \Gamma\left(\sigma_{M_{T}}^{2}-\sigma_{M_{T \mid t}}^{2}\right)\right]
\end{aligned}
$$

The term $\left(\sigma_{M_{T}}^{2}-\sigma_{M_{T \mid t}}^{2}\right)$ is a deterministic function of time, due to our assumptions about Gaussian interest rates and market prices of risk being constant or - at most - deterministic functions of time. The optimal wealth $W_{t}$ at any point in time $t \in(0, T)$ can be stated, cf. (62), as

$$
W_{t}=W_{0} \cdot M_{t}^{-\frac{1}{\gamma}} \cdot\left(\frac{D(t, T)}{D(0 ; T)}\right)^{\Gamma} \cdot \exp \left[\frac{1}{2 \gamma}\left(\sigma_{M_{T}}^{2}-\sigma_{M_{T \mid t}}^{2}\right)\right]
$$

From here the dynamics of the optimal wealth can be determined. To find the optimal portfolio it is only necessary to keep track of the risky part of this dynamics:

$$
d W_{t}=W_{t} \cdot\left[(\ldots) d t+\Gamma d D(t ; T)+\left(\frac{1}{\gamma}\right) \lambda^{\prime} d Z_{t}\right]
$$

Hence, the optimal portfolio has two components. One component mimics exactly the price development in the bond $D(t ; T)$ and enters with the weight $\Gamma=1-\frac{1}{\gamma}$. The other component is identical in form to the risky portfolio found under interest rate certainty, cf. (69), q.e.d. 


\section{B Proofs of Theorems 2 and 3 using truncated log-normally distributed random variables}

The proofs of theorem 2 and Theorem 3 are based on the following proposition concerning truncated log-normally distributed variables.

Proposition 2 Let $X$ and $Y$ be log-normally distributed and $(\log X, \log Y) \simeq N(\mu, \Sigma)$ where

$$
\mu=\left(\mu_{X}, \mu_{Y}\right), \Sigma=\left(\begin{array}{cc}
\sigma_{X}^{2} & \sigma_{X Y} \\
\sigma_{X Y} & \sigma_{Y}^{2}
\end{array}\right)
$$

Then the following four relations are valid:

$$
\begin{aligned}
P\{X>K\} & =N\left(\frac{-\log (K)+\mu_{X}}{\sigma_{X}}\right) \\
E\left[X 1_{\{X>K\}}\right] & =E[X] N\left(\frac{-\log (K)+\mu_{X}}{\sigma_{X}}+\sigma_{X}\right) \\
E\left[Y 1_{\{X>K\}}\right] & =E[Y] N\left(\frac{-\log (K)+\mu_{X}}{\sigma_{X}}+\frac{\sigma_{X Y}}{\sigma_{X}}\right) \\
E\left[X Y 1_{\{X>K\}}\right] & =E[X Y] N\left(\frac{-\log (K)+\mu_{X}}{\sigma_{X}}+\frac{\sigma_{X Y}}{\sigma_{X}}+\sigma_{X}\right)
\end{aligned}
$$

Proof These relations are proved in the appendix of Rubinstein (1976) and can also be found in chapter 6 of Huang and Litzenberger (1988).

$\underline{\text { Proof of Theorem } 2 \text { (continued) }}$

Let $X \equiv W_{T}$ and $Y \equiv W_{T}^{1-\gamma}$. Hence, using (i) in Theorem 1:

$$
\begin{gathered}
\mu_{X}=\log \left(W_{0}\right)+y(0 ; T) T+\left(\frac{1}{\gamma}-\frac{1}{2 \gamma^{2}}\right) \sigma_{M_{T}}^{2} \\
\sigma_{X}=\frac{1}{\gamma} \sigma_{M_{T}}, \quad \sigma_{Y}=\frac{1-\gamma}{\gamma} \sigma_{M_{T}}, \quad \sigma_{X Y}=\frac{1-\gamma}{\gamma^{2}} \sigma_{M_{T}}^{2} \quad \frac{\sigma_{X Y}}{\sigma_{X}}=\frac{1-\gamma}{\gamma} \sigma_{M_{T}}
\end{gathered}
$$

We use (80) to obtain the first term in (22):

$$
\begin{gathered}
P\left\{W_{T} \leq K\right\}=1-P\left\{W_{T}>K\right\}= \\
1-N\left(\frac{-\log (K)+\log \left(W_{0}\right)+y(0 ; T) T+\left(\frac{1}{\gamma}-\frac{1}{2 \gamma^{2}}\right) \sigma_{M_{T}}^{2}}{\frac{1}{\gamma} \sigma_{M_{T}}}\right)=1-N\left(-d_{1}^{*}\right)=N\left(d_{1}^{*}\right)
\end{gathered}
$$

Next use (82) to obtain the last term in (22): 


$$
\begin{gathered}
E_{0}\left[\frac{\left(x W_{T}\right)^{1-\gamma}}{1-\gamma} 1_{\left\{W_{T}>K\right\}}\right]= \\
J_{0}\left(x W_{0} ; T, \gamma\right) \cdot N\left(\frac{-\log (K)+\log \left(W_{0}\right)+y(0 ; T) T+\left(\frac{1}{\gamma}-\frac{1}{2 \gamma^{2}}\right) \sigma_{M_{T}}^{2}}{\frac{1}{\gamma} \sigma_{M_{T}}}+\frac{1-\gamma}{\gamma} \sigma_{M_{T}}\right)= \\
J_{0}\left(x W_{0} ; T, \gamma\right) \cdot N\left(\frac{(y(0 ; T)-g) T+\log (x)}{\frac{1}{\gamma} \sigma_{M_{T}}}+\frac{1}{2 \gamma} \sigma_{M_{T}}\right)=J_{0}\left(x W_{0} ; T, \gamma\right) \cdot\left(1-N\left(d_{2}^{*}\right)\right)
\end{gathered}
$$

q.e.d.

Proof of Theorem 3

Let $X \equiv \frac{W_{T}}{W_{0}}$ and $Y \equiv M_{T}$. Hence

$$
\begin{gathered}
\mu_{X}=y(0 ; T) T+\left(\frac{1}{\gamma}-\frac{1}{2 \gamma^{2}}\right) \sigma_{M_{T}}^{2} \\
\sigma_{Y}=\sigma_{M_{T}}, \quad \sigma_{X}=\frac{1}{\gamma} \sigma_{M_{T}}, \quad \sigma_{X Y}=-\frac{1}{\gamma} \sigma_{M_{T}}^{2} \\
P\left(W_{0}, g, T, x\right)=E_{0}\left[M_{T}\left(W_{0} e^{g T}-x W_{T}\right)^{+}\right]=x W_{0} \cdot E_{0}\left[\left(M_{T} e^{g T-\log (x)}-M_{T} \frac{W_{T}}{W_{0}}\right)^{+}\right]= \\
x W_{0} \cdot\left[E_{0}\left(M_{T} e^{g T-\log (x)} 1_{\left\{\frac{W_{T}}{W_{0}}<e^{g T-\log (x)}\right\}}\right)-E_{0}\left(M_{T} \frac{W_{T}}{W_{0}} 1_{\left\{\frac{W_{T}}{W_{0}}<e^{g T-\log (x)}\right\}}\right)\right]= \\
W_{0} e^{g T} E_{0}\left[M_{T}\left(1-1_{\left\{\frac{W_{T}}{W_{0}}>e^{g T-\log (x)}\right\}}\right)\right]-x W_{0} E_{0}\left[M_{T} \frac{W_{T}}{W_{0}}\left(1-1_{\left\{\frac{W_{T}}{W_{0}}>e^{g T-\log (x)}\right\}}\right)\right]=
\end{gathered}
$$

The two terms are found by inserting from the general results in Proposition 2 and using the symmetry of the normal distribution, $1-N(d)=N(-d)$ :

$$
\begin{gathered}
W_{0} e^{g T} E_{0}\left[M_{T}\left(1-1_{\left\{\frac{W_{T}}{W_{0}}>e^{g T-\log (x)}\right\}}\right)\right]= \\
W_{0} e^{g T} D(0 ; T) \cdot\left(1-N\left(\frac{\log (x)-g T+y(0 ; T) T+\left(\frac{1}{\gamma}-\frac{1}{2 \gamma^{2}}\right) \sigma_{M_{T}}^{2}}{\frac{1}{\gamma} \sigma_{M_{T}}}-\sigma_{M_{T}}\right)\right)= \\
W_{0} e^{g T} D(0 ; T) N\left(d_{1}^{*}+\sigma_{M_{T}}\right) \\
x W_{0} \cdot\left(1-N\left(\frac{\log (x)-g T+y(0 ; T) T+\left(\frac{1}{\gamma}-\frac{1}{2 \gamma^{2}}\right) \sigma_{M_{T}}^{2}}{x W_{0} E_{0}\left[M_{T} \frac{W_{T}}{W_{0}}\left(1-1_{\left\{\frac{W_{T}}{W_{0}}>e^{g T-\log (x)}\right\}}\right)\right]}=\right.\right. \\
\left.\left.\frac{1}{\gamma} \sigma_{M_{T}}+\frac{1}{\gamma} \sigma_{M_{T}}\right)\right) \\
x W_{0} N\left(d_{2}^{*}\right)
\end{gathered}
$$

q.e.d. 


\section{Comparative statics}

Equation (36):

In (36) the partial derivatives are easily worked out:

$$
\begin{aligned}
\frac{\partial \widetilde{J}_{0}}{\partial g} & =T\left(e^{g T}\right)^{1-\gamma} N\left(d_{1}^{*}\right) \\
\frac{\partial \widetilde{J}_{0}}{\partial x} & =\left(\frac{x}{D(0 ; t)} e^{\frac{1}{2 \gamma} \sigma_{M_{T}}^{2}}\right)^{1-\gamma} \frac{1}{x}\left(1-N\left(d_{2}^{*}\right)\right)
\end{aligned}
$$

Upon substituting for $\partial x / \partial g$ from (32) the terms in brackets in (36) reduce to

$$
\begin{aligned}
\frac{\partial \widetilde{J}_{0}}{\partial g}+\frac{\partial \widetilde{J}_{0}}{\partial x} \frac{\partial x}{\partial g} & =\left[T\left(e^{g T}\right)^{1-\gamma} N\left(d_{1}^{*}\right)-\left(\frac{x}{D(0 ; t)} e^{\frac{1}{2 \gamma} \sigma_{M_{T}}^{2}}\right)^{-\gamma} T e^{\left.g T+\frac{1}{2 \gamma} \sigma_{M_{T}}^{2} N\left(d_{1}^{*}+\sigma_{M_{T}}\right)\right]}\right. \\
& =T e^{g T}\left[\left(e^{g T}\right)^{-\gamma} N\left(d_{1}^{*}\right)-\left(\frac{x}{D(0 ; t)} e^{\frac{1}{2 \gamma} \sigma_{M_{T}}^{2}}\right)^{-\gamma} e^{\left.\frac{1}{2 \gamma} \sigma_{M_{T}}^{2} N\left(d_{1}^{*}+\sigma_{M_{T}}\right)\right]}\right.
\end{aligned}
$$

Applying Proposition 2 this can be written as

$$
-T e^{g T} E_{0}\left[\left(\left[x W_{T}\right]^{-\gamma}-\left[e^{g T}\right]^{-\gamma}\right)^{+}\right]<0
$$

which is obviously negative.

Equation (37):

The calculations in (37) are more involved. First we calculate the latter term within parenthesis.

$$
\frac{\partial \widetilde{J}_{0}}{\partial x} \frac{\partial x}{\partial \gamma}=\left(\frac{x}{D(0 ; T)} e^{\frac{1}{2 \gamma} \sigma_{M_{T}}^{2}}\right)^{1-\gamma} \cdot\left(1-N\left(d_{2}^{*}\right)\right) \cdot \frac{\partial x}{\partial \gamma} \frac{1}{x}
$$

Inserting from (33) leads to

$$
\frac{\partial \widetilde{J}_{0}}{\partial x} \frac{\partial x}{\partial \gamma}=\left(\frac{x}{D(0 ; T)} e^{\frac{1}{2 \gamma} \sigma_{M_{T}}^{2}}\right)^{1-\gamma} N^{\prime}\left(d_{2}^{*}\right) \frac{\sigma_{M_{T}}}{\gamma^{2}}>0
$$

The $\gamma$-terms involve a number of complex expressions. Observe that by construction we have

$$
\widetilde{J}_{0}(1 ; T, \gamma)-J_{0}\left(\widehat{W}_{0} ; T, \gamma\right)=0
$$

Next observe that

$$
e^{g T(1-\gamma)} N^{\prime}\left(d_{1}^{*}\right)=\left(\frac{x}{D(0 ; T)} e^{\frac{1}{2 \gamma} \sigma_{M_{T}}^{2}}\right)^{1-\gamma} N^{\prime}\left(d_{2}^{*}\right)
$$


and that

$$
d_{1}^{*}=d_{2}^{*}-\Gamma \sigma_{M_{T}} \Rightarrow \frac{\partial d_{1}^{*}}{\partial \gamma}=\frac{\partial d_{2}^{*}}{\partial \gamma}-\frac{1}{\gamma^{2}} \sigma_{M_{T}}
$$

Hence, using these relations and brute force calculations we end up with the following expres$\operatorname{sion}^{10}$

$$
\begin{aligned}
& (1-\gamma) \cdot\left(\frac{\partial \widetilde{J}_{0}}{\partial \gamma}-\frac{\partial J_{0}}{\partial \gamma}+\frac{\partial \widetilde{J}_{0}}{\partial x} \frac{\partial x}{\partial \gamma}\right)= \\
& \left(\frac{x}{D(0 ; T)} e^{\frac{1}{2 \gamma} \sigma_{M_{T}}^{2}}\right)^{1-\gamma} \cdot\left(1-N\left(d_{2}^{*}\right)\right) \cdot[(g-y(0 ; T)) T-\log (x)] \\
& -\left(\frac{\widehat{W_{0}}}{D(0 ; T)} e^{\frac{1}{2 \gamma} \sigma_{M_{T}}^{2}}\right)^{1-\gamma} \cdot\left[-\log \left(\widehat{W_{0}}\right)+(g-y(0 ; T)) T\right] \\
& -\left(\frac{x}{D(0 ; T)} e^{\frac{1}{2 \gamma} \sigma_{M_{T}}^{2}}\right)^{1-\gamma} \cdot N^{\prime}\left(d_{2}^{*}\right) \cdot \frac{1}{\gamma^{2}} \sigma_{M_{T}} \\
& +(1-\gamma)\left(\frac{x}{D(0 ; T)} e^{\frac{1}{2 \gamma} \sigma_{M_{T}}^{2}}\right)^{1-\gamma} \cdot N^{\prime}\left(d_{2}^{*}\right) \cdot \frac{1}{\gamma^{2}} \sigma_{M_{T}} \\
& {\left[\left(\frac{\widehat{W_{0}}}{D(0 ; T)} e^{\frac{1}{2 \gamma} \sigma_{M_{T}}^{2}}\right)^{1-\gamma}-\left(\frac{x}{D(0 ; T)} e^{\frac{1}{2 \gamma} \sigma_{M_{T}}^{2}}\right)^{1-\gamma} \cdot\left(1-N\left(d_{2}^{*}\right)\right)\right] \cdot \frac{1}{2 \gamma^{2}} \sigma_{M_{T}}^{2}}
\end{aligned}
$$

By collecting terms we end up with the expression

$$
\begin{aligned}
& (1-\gamma) \cdot\left(\frac{\partial \widetilde{J}_{0}}{\partial \gamma}-\frac{\partial J_{0}}{\partial \gamma}+\frac{\partial \widetilde{J}_{0}}{\partial x} \frac{\partial x}{\partial \gamma}\right)= \\
& {\left[\left(\frac{\widehat{W_{0}}}{D(0 ; T)} e^{\frac{1}{2 \gamma} \sigma_{M_{T}}^{2}}\right)^{1-\gamma}-\left(\frac{x}{D(0 ; T)} e^{\frac{1}{2 \gamma} \sigma_{M_{T}}^{2}}\right)^{1-\gamma} \cdot\left(1-N\left(d_{2}^{*}\right)\right)\right]} \\
& {\left[(y(0 ; T)-g) T+\log \left(\widehat{W}_{0}\right)+\frac{1}{2 \gamma^{2}} \sigma_{M_{T}}^{2}\right]+} \\
& \left(\frac{x}{D(0 ; T)} e^{\frac{1}{2 \gamma} \sigma_{M_{T}}^{2}}\right)^{1-\gamma} \cdot\left(1-N\left(d_{2}^{*}\right)\right) \cdot\left[\log \left(\widehat{W}_{0}\right)-\log (x)\right] \\
& -\left(\frac{x}{D(0 ; T)} e^{\frac{1}{2 \gamma} \sigma_{M_{T}}^{2}}\right)^{1-\gamma} \cdot N^{\prime}\left(d_{2}^{*}\right) \cdot \frac{1}{\gamma} \sigma_{M_{T}}
\end{aligned}
$$

\footnotetext{
${ }^{10}$ For ease of notation we multiply the final expression with $1-\gamma$.
} 